\title{
VOLUME OF MIXED BODIES
}

\author{
BY
}

\author{
ERWIN LUTWAK
}

\begin{abstract}
By using inequalities obtained for the volume of mixed bodies and the Petty Projection Inequality, (sharp) isoperimetric inequalities are derived for the projection measures (Quermassintegrale) of a convex body. These projection measure inequalities, which involve mixed projection bodies (zonoids), are shown to be strengthened versions of the classical inequalities between the projection measures of a convex body. The inequality obtained for the volume of mixed bodies is also used to derive a form of the Brunn-Minkowski inequality involving mixed bodies. As an application, inequalities are given between the projection measures of convex bodies and the mixed projection integrals of the bodies.
\end{abstract}

0. Introduction. In [24] it was shown that the Petty projection inequality [26] will yield extensions of the known isoperimetric inequalities between the volume of a convex body and its projection measures (Quermassintegrale). In this article we show that the Petty projection inequality can be used to obtain (sharp) isoperimetric inequalities (Corollary (5.20)) which are strengthened forms of the classical inequalities between the projection measures of a convex body. This is accomplished by using the so-called mixed convex bodies introduced by Firey [17]. Geometric inequalities are derived for the volume of mixed bodies (Theorems (4.1) and (4.3)) which, when combined with the Petty projection inequality, yield inequalities involving projection bodies (zonoids) which are related to the inequalities between the projection measures of a convex body. The inequalities for mixed bodies are also used to obtain a version of the Brunn-Minkowski inequality (Theorem (4.2)) involving mixed bodies. Also derived are inequalities between the volume of convex bodies and their associated mixed projection integral (Theorems (6.4) and (6.4*)), that are extensions and improvements of some previously established inequalities.

Since some of our results concern projection bodies, a topic of interest to workers outside the area of geometric convexity, we have restated a few basic definitions and standard results from the field of geometric inequalities. In $\$ 1$ we collect for quick reference some well-known results concerning mixed volumes and mixed area measures which will be required later. References for material presented in this section are Bonnesen and Fenchel [7] (for mixed volumes), Busemann [9], and Leichtweiss [20]. The original works of Aleksandrov [1-4] and Fenchel and Jessen [15], supplemented by the survey of Schneider [28], are recommended references for mixed area measures. $\$ 2$ contains introductory material regarding Blaschke addition.

Received by the editors January 8, 1985.

1980 Mathematics Subject Classification. Primary 52A40; Secondary 53A15, 46B20.

Key words and phrases. Convex body, mixed area measure, mixed body, mixed volume, projection body, projection measure (Quermassintegrale), zonoid. 
(For a nice application of Blaschke addition, see Firey and Grünbaum [16], or Grübaum [18, Chapter 15.3].) Mixed bodies are introduced in $\S 3$ where a few basic properties are listed. Nothing in $\S \S 2$ and 3 is original. Most of the material presented is that of Firey [17], which is the recommended reference for both sections. In $\S 4$ we examine the volume of mixed bodies. Here inequalities are obtained for the volume of mixed bodies (Theorems (4.1) and (4.3) and Proposition (4.4)). As an application, a version of the Brunn-Minkowski inequality (Theorem (4.2)) involving mixed bodies is given. One of the upper bounds for the volume of the mixed body of a convex body and a ball, obtained in $\S 4$ by using the Minkowski inequality, can be significantly improved (inequality (5.13)) by appealing to the Petty projection inequality. This is accomplished in $§ 5$. Here, new isoperimetric inequalities (Theorem (5.16)) for the volume of mixed projection bodies are obtained which are improvements of some similar inequalities obtained in [24]. Recommended references for projection bodies are the surveys by Bolker [6] and Schneider and Weil [30]. A good reference regarding mixed brightness and girth functions, which are considered in $\S 5$, is Chakerian [10]. In $\S 6$ inequalities involving mixed projection integrals are presented. The mixed projection integrals (introduced in $[\mathbf{2 3}, \mathbf{2 4}]$ ) are similar to means considered previously by Chakerian [11-13], Chakerian and Sangwine-Yager [14] and the author [21, 22] (see also Burago and Zalgaller [8, pp. 170-171]). The inequalities (Theorems (6.4) and $\left(6.4^{*}\right)$ ) obtained here are improved versions of those derived by the author in [24].

1. Mixed volumes and mixed area measures. By a convex figure in Euclidean $n$-space, $\mathbb{R}^{n}$ ( $n>2$ is assumed throughout), we mean a compact convex subset of $\mathbb{R}^{n}$. The term convex body will be reserved for convex figures with interior points. The Minkowski sum of the convex figures $K_{1}, \ldots, K_{r}$ is defined by

$$
K_{1}+\cdots+K_{r}=\left\{x_{1}+\cdots+x_{r}: x_{1} \in K_{1}, \ldots, x_{r} \in K_{r}\right\} .
$$

For a convex figure $K$ and a nonnegative scalar $\lambda$ the Minkowski scalar product $\lambda K$ is $\{\lambda x: x \in K\}$. Associated with a convex figure $K$ is its support function, $h(K, \cdot)$, defined on $\mathbb{R}^{n}$ by

$$
h(K, x)=\operatorname{Max}\{x \cdot y: y \in K\},
$$

where $x \cdot y$ is the usual inner product of $x$ and $y$ in $\mathbb{R}^{n}$. If $K$ is a convex body that contains the origin in its interior, then we also associate with $K$ its radial function, $\rho(K, \cdot)$, defined on the unit sphere centered at the origin, $S^{n-1}$, by

$$
\rho(K, u)=\operatorname{Max}\{\lambda>0: \lambda u \in K\} .
$$

We shall use $B$ to denote the unit ball in $\mathbb{R}^{n}$ and $\omega_{n}$ to denote its volume.

If $K_{1}, \ldots, K_{r}$ are convex figures and $\lambda_{1}, \ldots, \lambda_{r}$ are nonnegative real numbers, then of fundamental importance is the fact that the volume of $\lambda_{1} K_{1}+\cdots+\lambda_{r} K_{r}$ is a homogeneous polynomial of degree $n$ in $\lambda_{1}, \ldots, \lambda_{r}$ given by

$$
V\left(\lambda_{1} K_{1}+\cdots+\lambda_{r} K_{r}\right)=\sum \lambda_{i_{1}} \cdots \lambda_{i_{n}} V\left(K_{i_{1}}, \ldots, K_{i_{n}}\right),
$$


where the sum is taken over all $n$-tuples of positive integers $\left(i_{1}, \ldots, i_{n}\right)$ whose entries do not exceed $r$. The coefficient $V\left(K_{i_{1}}, \ldots, K_{i_{n}}\right)$ is called the mixed volume of $K_{i_{1}}, \ldots, K_{i_{n}}$ and is uniquely determined by the requirement that it be symmetric in its arguments. If $s$ and $t$ are nonnegative integers whose sum does not exceed $n, K$ and $L$ are convex figures, and $\mathscr{C}$ is the $(n-s-t)$-tuple of convex figures $\left(C_{1}, \ldots, C_{n-s-t}\right)$, then $V(K, s ; L, t ; \mathscr{C})$ will be used to denote the mixed volume $V\left(K, \ldots, K, L, \ldots, L, C_{1}, \ldots, C_{n-s-t}\right)$ in which $K$ appears $s$ times and $L$ appears $t$ times. The mixed volume $V(K, n-i ; L, i)$ is usually written $V_{i}(K, L)$. If $L=B$, then $V_{i}(K, B)$ is just the $i$ th projection measure (Quermassintegral) of $K, W_{i}(K)$. We recall that $W_{0}(K)$ is $V(K), n W_{1}(K)$ is the surface area of $K, S(K)$, while $\left(2 / \omega_{n}\right) W_{n-1}(K)$ is the mean width of $K$.

The Aleksandrov-Fenchel inequality [3] (see also [9 or 20]), in the form most suitable for our purposes, states that

$$
V^{s}(K, s+t ; \mathscr{C}) V^{t}(L, s+t ; \mathscr{C}) \leqslant V^{s+t}(K, s ; L, t ; \mathscr{C}) .
$$

The equality conditions in the Aleksandrov-Fenchel inequality are unknown unless very restrictive assumptions are made on the bodies in question (see Schneider [29] for a discussion, conjectures, and partial results). A special case of this general inequality, for which the equality conditions are known, is the Minkowski inequality which states that if $K$ and $L$ are convex bodies in $\mathbb{R}^{n}$, then

$$
V^{n-1}(K) V(L) \leqslant V_{1}^{n}(K, L),
$$

with equality if and only if $K$ and $L$ are homothetic. If $K_{1}, \ldots, K_{n-1}$ are convex bodies in $\mathbb{R}^{n}$, then by repeated applications of the Aleksandrov-Fenchel inequalities, with final applications of the Minkowski inequality, one obtains

$$
V\left(K_{1}\right) \cdots V\left(K_{n}\right) \leqslant V^{n}\left(K_{1}, \ldots, K_{n}\right),
$$

with equality if and only if the $K_{i}$ are homothetic.

Associated with the convex bodies $K_{1}, \ldots, K_{n-1}$ in $\mathbb{R}^{n}$ is a unique (positive) Borel measure on $S^{n-1}, S\left(K_{1}, \ldots, K_{n-1} ; \cdot\right)$, called the mixed area measure of $K_{1}, \ldots, K_{n-1}$, with the property that for any convex figure $K$ in $\mathbb{R}^{n}$ one has the integral representation

$$
V\left(K_{1}, \ldots, K_{n-1}, K\right)=\frac{1}{n} \int_{S^{n-1}} h(K, u) d S\left(K_{1}, \ldots, K_{n-1} ; u\right),
$$

where the integration is with respect to the measure $S\left(K_{1}, \ldots, K_{n-1} ; \cdot\right)$ on $S^{n-1}$. The mixed area measure $S\left(K_{1}, \ldots, K_{n-1} ; \cdot\right)$ is symmetric in its (first $\left.n-1\right)$ arguments. It is invariant under translations of the $K_{i}$. If $K_{1}$ is replaced by $K_{1}+L_{1}$, then from the corresponding property for the mixed volumes and the uniqueness of the mixed area measure one has,

$$
S\left(K_{1}+L_{1}, K_{2}, \ldots, K_{n-1} ; \cdot\right)=S\left(K_{1}, \ldots, K_{n-1} ; \cdot\right)+S\left(L_{1}, K_{2}, \ldots, K_{n-1} ; \cdot\right) .
$$


From the corresponding property of the mixed volumes it also follows that

$$
S\left(\lambda_{1} K_{1}, \ldots, \lambda_{n-1} K_{n-1} ; \cdot\right)=\lambda_{1} \cdots \lambda_{n-1} S\left(K_{1}, \ldots, K_{n-1} ; \cdot\right) .
$$

The mixed area measure $S(K, \ldots, K, B, \ldots, B ; \cdot)$, with $i$ copies of $K$ and $n-i-1$ copies of $B$, is usually written $S_{i}(K, \cdot)$. The mixed area measure $S_{n-1}(K, \cdot)$ is called the surface area measure of $K$ and is denoted by $S(K, \cdot)$. For $K=B$, the surface area measure is just ordinary spherical Lebesgue measure on $S^{n-1}$, for which we write $S(\cdot)$ rather than $S(B, \cdot)$.

A uniqueness theorem of Aleksandrov [2] and Fenchel and Jessen [15], of which we make frequent use, states that if $K$ and $L$ are convex bodies in $\mathbb{R}^{n}$ such that, for some $i>0, S_{i}(K, \cdot)=S_{i}(L, \cdot)$, then $K$ and $L$ must be translates of each other. The theorem of Aleksandrov and Fenchel and Jessen is, in fact, more general than this (see, for example, [28, p. 43]). We shall, however, require only the stated version. This theorem often will be the basis for concluding the equality (up to translation) of two convex bodies. Throughout this article, a statement about the equality of two convex bodies should always be interpreted to mean equality up to translation.

An important fact (see, for example, [15, p. 24]) that we require about the mixed area measure $S\left(K_{1}, \ldots, K_{n-1} ; \cdot\right)$, of the convex bodies $K_{1}, \ldots, K_{n-1}$ in $\mathbb{R}^{n}$, is that the measure is not concentrated on any great sphere of $S^{n-1}$, and has the origin as its centroid (when viewed as a mass loading on $S^{n-1}$ ); i.e.,

$$
\int_{S^{n-1}} u d S\left(K_{1}, \ldots, K_{n-1} ; u\right)=0
$$

2. Blaschke sums. Minkowski's existence theorem (which in the form used here is due to Aleksandrov $[3,5]$ and Fenchel and Jessen [15]) states that corresponding to a (positive) Borel measure $\mu$ on $S^{n-1}$, which is not concentrated on any great sphere and which satisfies

$$
\int_{S^{n-1}} u d \mu(u)=0
$$

there exists a convex body $K$ in $\mathbb{R}^{n}$, unique up to translation, such that $\mu=S(K, \cdot)$.

If $K_{1}, K_{2}$ are convex bodies and $\lambda_{1}, \lambda_{2}$ are nonnegative scalars (not both 0 ), then since $S\left(K_{1}, \cdot\right)$ and $S\left(K_{2}, \cdot\right)$ satisfy the hypothesis of Minkowski's theorem, so does $\lambda_{1} S\left(K_{1}, \cdot\right)+\lambda_{2} S\left(K_{2}, \cdot\right)$. Hence, by Minkowski's theorem there exists a convex body, unique up to translation, which we denote by $\lambda_{1} \cdot K_{1}+\lambda_{2} \cdot K_{2}$, whose surface area measure is given by

$$
S\left(\lambda_{1} \cdot K_{1}+\lambda_{2} \cdot K_{2}, \cdot\right)=\lambda_{1} S\left(K_{1}, \cdot\right)+\lambda_{2} S\left(K_{2}, \cdot\right) .
$$

The addition and scalar multiplication defined in this manner are called Blaschke addition and scalar multiplication. From (1.6) and the uniqueness theorem of Aleksandrov and Fenchel and Jessen, one sees that the relation between Blaschke and Minkowski scalar multiplication is

$$
\lambda \cdot K=\lambda^{1 /(n-1)} K
$$


It is trivial to verify that Blaschke addition is both commutative and associative and that

$$
\begin{aligned}
1 \cdot K & =K, \\
\lambda \cdot\left(K_{1}+K_{2}\right) & =\lambda \cdot K_{1}+\lambda \cdot K_{2}, \\
\left(\lambda_{1} \lambda_{2}\right) \cdot K & =\lambda_{1} \cdot\left(\lambda_{2} \cdot K\right), \\
\left(\lambda_{1}+\lambda_{2}\right) \cdot K & =\lambda_{1} \cdot K+\lambda_{2} \cdot K .
\end{aligned}
$$

From (1.4) and the definition of Blaschke addition and scalar multiplication it follows that for convex bodies $K_{1}, K_{2}, C$, and nonnegative scalars $\lambda_{1}, \lambda_{2}$, one has

$$
V_{1}\left(\lambda_{1} \cdot K_{1}+\lambda_{2} \cdot K_{2}, C\right)=\lambda_{1} V_{1}\left(K_{1}, C\right)+\lambda_{2} V_{1}\left(K_{2}, C\right)
$$

3. Mixed bodies. It has already been noted that the mixed area measure $S\left(K_{1}, \ldots, K_{n-1} ; \cdot\right)$ satisfies the hypothesis of Minkowski's existence theorem. Thus, corresponding to the convex bodies $K_{1}, \ldots, K_{n-1}$ in $\mathbb{R}^{n}$, there exists a convex body, unique up to translation, which we denote by $\left[K_{1}, \ldots, K_{n-1}\right]$, whose area function is $S\left(K_{1}, \ldots, K_{n-1} ; \cdot\right)$; i.e.,

$$
S\left(\left[K_{1}, \ldots, K_{n-1}\right], \cdot\right)=S\left(K_{1}, \ldots, K_{n-1} ; \cdot\right) .
$$

From the (previously stated) properties of mixed area measures and the uniqueness theorem of Aleksandrov and Fenchel and Jessen it follows that $\left[K_{1}, \ldots, K_{n-1}\right]$ is symmetric in its arguments, and that if the $K_{i}$ are replaced by homothetic copies, the resulting mixed body will be homothetic to the original. It also follows that $[K, \ldots, K]=K$, where, as previously noted, such equalities are assumed to mean up to translation.

From (1.5), (1.6), definition (2.1), and the uniqueness theorem of Aleksandrov and Fenchel and Jessen, it follows that,

$$
\left[K_{1}+L_{1}, K_{2}, \ldots, K_{n-1}\right]=\left[K_{1}, K_{2}, \ldots, K_{n-1}\right]+\left[L_{1}, K_{2}, \ldots, K_{n-1}\right],
$$

and that

$$
\left[\lambda_{1} K_{1}, \ldots, \lambda_{n-1} K_{n-1}\right]=\left(\lambda_{1} \cdots \lambda_{n-1}\right) \cdot\left[K_{1}, \ldots, K_{n-1}\right] .
$$

As an immediate consequence of (1.4) and the definition of a mixed body one has

$$
V_{1}\left(\left[K_{1}, \ldots, K_{n-1}\right], K_{n}\right)=V\left(K_{1}, \ldots, K_{n-1}, K_{n}\right) .
$$

For the mixed body $[K, \ldots, K, L, \ldots, L]$, with $i$ copies of $L$ and $n-i-1$ copies of $K$, we write $[K, L]_{i}$. For the case where $L=B$, we write $[K]_{i}$ rather than $[K, B]_{i}$. We note that $[K]_{0}=K$, while $[K]_{n-1}=B$.

Obviously $K+L=[K+L, \ldots, K+L]$. If we use (3.2), (2.2) and the fact that a mixed body is symmetric in its arguments, we get

$$
K+L=\sum_{i=0}^{n-1}\left(\begin{array}{c}
n-1 \\
i
\end{array}\right) \cdot[K, L]_{i},
$$

where the sum on the right denotes a Blaschke sum. This was obtained by Firey [17] using a slightly different approach. 
4. Volume of mixed bodies. If $K_{1}, \ldots, K_{n-1}$ are convex bodies in $\mathbb{R}^{n}$, then since

$$
V\left(\left[K_{1}, \ldots, K_{n-1}\right]\right)=V_{1}\left(\left[K_{1}, \ldots, K_{n-1}\right],\left[K_{1}, \ldots, K_{n-1}\right]\right),
$$

we can use (3.4) to conclude that

$$
V\left(\left[K_{1}, \ldots, K_{n-1}\right]\right)=V\left(K_{1}, \ldots, K_{n-1},\left[K_{1}, \ldots, K_{n-1}\right]\right) .
$$

If we use inequality (1.3) with $K_{n}=\left[K_{1}, \ldots, K_{n-1}\right]$, we get

(4.1) ThEOREM. If $K_{1}, \ldots, K_{n-1}$ are convex bodies in $\mathbb{R}^{n}$, then

$$
V\left(K_{1}\right) \cdots V\left(K_{n-1}\right) \leqslant V^{n-1}\left(\left[K_{1}, \ldots, K_{n-1}\right]\right),
$$

with equality if and only if the $K_{i}$ are homothetic.

As an aside, we note that from the Aleksandrov-Fenchel inequality (or the isepiphanic inequality) one easily obtains an inequality which gives an upper bound for the volume for $\left[K_{1}, \ldots, K_{n-1}\right]$ :

$$
\omega_{n} V\left(\left[K_{1}, \ldots, K_{n-1}\right]\right)^{n-1} \leqslant V^{n}\left(K_{1}, \ldots, K_{n-1}, B\right) .
$$

We note, also as an aside, that from the Aleksandrov-Fenchel inequality, it is easy to obtain the counterpart of Theorem (4.1) for surface area rather than volume:

$$
S\left(K_{1}\right) \cdots S\left(K_{n-1}\right) \leqslant S\left(\left[K_{1}, \ldots, K_{n-1}\right]\right)^{n-1} .
$$

The Brunn-Minkowski inequality states that if $K$ and $L$ are convex bodies in $\mathbb{R}^{n}$, then

$$
V^{1 / n}(K)+V^{1 / n}(L) \leqslant V^{1 / n}(K+L),
$$

with equality if and only if $K$ and $L$ are homothetic. From Theorem (4.1) we obtain

(4.2) THEOREM. If $K$ and $L$ are convex bodies in $\mathbb{R}^{n}$, then

$$
V^{1 / n}(K)+V^{1 / n}(L) \leqslant\left[\sum_{i=0}^{n-1}\left(\begin{array}{c}
n-1 \\
i
\end{array}\right) V\left([K, L]_{i}\right)^{(n-1) / n}\right]^{1 /(n-1)} \leqslant V^{1 / n}(K+L)
$$

with equality, in either of these inequalities, if and only if $K$ and $L$ are homothetic.

Proof. Suppose $C$ is an arbitrary convex body. From (3.5) we have

$$
V_{1}(K+L, C)=V_{1}\left(\sum_{i=0}^{n-1}\left(\begin{array}{c}
n-1 \\
i
\end{array}\right) \cdot[K, L]_{i}, C\right) \text {, }
$$

and, hence, from (2.3) we get

$$
V_{1}(K+L, C)=\sum_{i=0}^{n-1}\left(\begin{array}{c}
n-1 \\
i
\end{array}\right) V_{1}\left([K, L]_{i}, C\right) .
$$

The Minkowski inequality (1.2) can now be used to conclude that

$$
V_{1}(K+L, C) \geqslant \sum_{i=0}^{n-1}\left(\begin{array}{c}
n-1 \\
i
\end{array}\right) V\left([K, L]_{i}\right)^{(n-1) / n} V(C)^{1 / n}
$$


with equality if and only if $[K, L]_{i}$ is homothetic to $C$ for $i=0,1, \ldots, n-1$. For equality to occur it is necessary that $[K, L]_{0}=K$ and $[K, L]_{n-1}=L$ both be homothetic to $C$. However, the requirement that $K$ and $L$ be homothetic to $C$ is sufficient to conclude that $[K, L]_{i}$ is homothetic to $C$ for $i=0,1, \ldots, n-1$. If we now take $C$ to be $K+L$, our last inequality becomes

$$
V(K+L)^{(n-1) / n} \geqslant \sum_{i=0}^{n-1}\left(\begin{array}{c}
n-1 \\
i
\end{array}\right) V\left([K, L]_{i}\right)^{(n-1) / n}
$$

with equality if and only if $K$ and $L$ are homothetic. From Theorem (4.1) it follows that

$$
\sum_{i=0}^{n-1}\left(\begin{array}{c}
n-1 \\
i
\end{array}\right) V\left([K, L]_{i}\right)^{(n-1) / n} \geqslant \sum_{i=0}^{n-1}\left(\begin{array}{c}
n-1 \\
i
\end{array}\right) V(K)^{(n-i-1) / n} V(L)^{i / n}
$$

with equality if and only if $K$ and $L$ are homothetic. Since the right side of this inequality is just

$$
\left(V(K)^{1 / n}+V(L)^{1 / n}\right)^{n-1}
$$

the proof is complete.

For convex bodies $K, L$ in $\mathbb{R}^{n}$, the Kneser-Süss inequality (see [7, p. 124]) states that

$$
V(K)^{(n-1) / n}+V(L)^{(n-1) / n} \leqslant V(K+L)^{(n-1) / n}
$$

with equality if and only if $K$ and $L$ are homothetic. We observe that if one were to start with the Kneser-Süss inequality and Theorem (4.1), then by applying the Kneser-Süss inequality to (3.5) and then using Theorem (4.1), one would immediately get the Brunn-Minkowski inequality, along with the conditions for equality.

A better result than Theorem (4.1) is possible for the case where $K_{1}=\cdots=$ $K_{n-i-1}=K$ and $K_{n-i}=\cdots=K_{n-1}=B$ :

(4.3) TheOREM. If $K$ is a convex body in $\mathbb{R}^{n}$ and $0<i<n-1$, then

$$
\omega_{n}^{i / n(n-i)} W_{i}(K)^{(n-i-1) /(n-i)} \leqslant V\left([K]_{i}\right)^{(n-1) / n} \leqslant \omega_{n}^{-1 / n} W_{i+1}(K),
$$

with equality, in either inequality, if and only if $K$ is a ball.

Proof. Since $V\left([K]_{i}\right)=V_{1}\left([K]_{i},[K]_{i}\right)$, from (3.4) it follows that

$$
V\left([K]_{i}\right)=V\left(K, n-i-1 ; B, i ;[K]_{i}\right) \text {. }
$$

The Aleksandrov-Fenchel inequality (1.1), with $L=[K]_{i}, s=n-i-1$ and $t=1$, can be used to conclude that

$$
V\left([K]_{i}, n-i ; B, i\right) V(K, n-i ; B, i)^{n-i-1} \leqslant V\left([K]_{i}\right)^{n-i} .
$$

However, from (1.3) we have

$$
\omega_{n}^{i / n} V\left([K]_{i}\right)^{(n-i) / n} \leqslant V\left([K]_{i}, n-i ; B, i\right),
$$


with equality if and only if $[K]_{i}$ is a ball. If we combine the two inequalities above, we obtain the left inequality of our theorem. To obtain the right inequality we combine the observation that

$$
W_{i+1}(K)=V(K, n-i-1 ; B, i ; B)
$$

with (3.4) to conclude that

$$
W_{i+1}(K)=V_{1}\left([K]_{i}, B\right) .
$$

The Minkowski inequality (1.2) will then yield

$$
\omega_{n} V\left([K]_{i}\right)^{n-1} \leqslant W_{i+1}(K)^{n},
$$

with equality if and only if $[K]_{i}$ is a ball. This is the right inequality of our theorem. In both of the inequalities of our theorem equality occurs if and only if $[K]_{i}$ is a ball, or equivalently, if and only if $S_{n-i-1}(K, \cdot)=S_{n-i-1}\left(B^{\prime}, \cdot\right)$ for some ball $B^{\prime}$. Since $i<n-1$, the uniqueness theorem of Aleksandrov and Fenchel and Jessen shows that this is possible if and only if $K$ itself is a ball.

We observe that the inequality between the left and right quantities in the inequality of Theorem (4.3) is the well-known inequality between consecutive projection measures of a convex body (see, for example, Aleksandrov [2]).

Before stating our next result we recall that if $K$ and $L$ are convex bodies in $\mathbb{R}^{n}$, then $K$ is called an $i$-tangential body of $L$ if $L \subset K$ and every $(n-i-1)$-extremal support hyperplane of $K$ is also a support hyperplane of $L$.

(4.4) Proposition. If $K$ is a convex body in $\mathbb{R}^{n}$ and $0<i<n-1$, then

$$
V\left([K]_{i}\right)^{n-1} \leqslant W_{i}^{n}(K) / V(K),
$$

with equality if and only if $K$ is an $(n-i-1)$-tangential body of a ball.

Proof. Since $W_{i}(K)=V(K, n-i-1 ; B, i ; K)$, it follows from (3.4) that $W_{i}(K)$ $=V_{1}\left([K]_{i}, K\right)$. From the Minkowski inequality we get $V\left([K]_{i}\right)^{n-1} V(K) \leqslant W_{i}^{n}(K)$, with equality if and only if $[K]_{i}$ and $K$ are homothetic. The conditions for equality reduce to $S_{n-i-1}(K, \cdot)=\lambda S(K, \cdot)$ for some positive $\lambda$, and Schneider [27] has proven that this is possible if and only if $K$ is an $(n-i-1)$-tangential body of a ball.

5. Mixed projection bodies. If $K$ is a convex body in $\mathbb{R}^{n}$ and $u \in S^{n-1}$, then we use $K^{u}$ to denote the orthogonal projection of $K$ onto the hyperplane $E_{u}$ that passes through the origin and is orthogonal to $u$. If $K_{1}, \ldots, K_{n-1}$ are convex bodies in $\mathbb{R}^{n}$ and $u \in S^{n-1}$, then the $(n-1)$-dimensional mixed volume of $K_{1}^{u}, \ldots, K_{n-1}^{u}$ in $E_{u}$ is written $v\left(K_{1}^{u}, \ldots, K_{n-1}^{u}\right)$ and called the mixed brightness of $K_{1}, \ldots, K_{n-1}$ in the direction $u$. If $K_{1}=\cdots=K_{n-i-1}=K$ and $K_{n-i}=\cdots=K_{n-1}=B$, then the mixed volume $v\left(K_{1}^{u}, \ldots, K_{n-1}^{u}\right)$ is just the $i$ th projection measure of $K^{u}$ in $E_{u}$; it will be denoted by $w_{i}\left(K^{u}\right)$ and is called the $(n-i-1)$-girth of $K$ in the direction $u$. The $(n-1)$-dimensional volume of $K^{u}$ will be written $v\left(K^{u}\right)$, rather than $w_{0}\left(K^{u}\right)$, and is called the brightness of $K$ in the direction $u$. 
The projection body of a convex body $K, \Pi K$, is the centrally symmetric body whose support function on $S^{n-1}$ is given by

$$
h(\Pi K, u)=v\left(K^{u}\right) .
$$

If $K_{1}, \ldots, K_{n-1}$ are convex bodies in $\mathbb{R}^{n}$, then the mixed projection body of $K_{1}, \ldots, K_{n-1}$ is the centrally symmetric body (see, for example, [7 or 24]) whose support function on $S^{n-1}$ is given by

$$
h\left(\Pi\left(K_{1}, \ldots, K_{n-1}\right), u\right)=v\left(K_{1}^{u}, \ldots, K_{n-1}^{u}\right) .
$$

We note that $\Pi(K, \ldots, K)=\Pi K$.

The mixed projection body $\Pi(K, \ldots, K, L, \ldots, L)$, with $i$ copies of $L$ and $n-i-1$ copies of $K$, will be denoted by $\Pi_{i}(K, L)$. $\Pi_{i} K=\Pi_{i}(K, B)$ is the $i$ th projection body of $K$. (We note that our indexing differs from that used in [30].) From our definition of $\Pi_{i} K$ it follows that

$$
h\left(\Pi_{i} K, u\right)=w_{i}\left(K^{u}\right),
$$

and $\Pi_{0} K=\Pi K$.

If $K$ is a convex body that contains the origin in its interior, we will write $K^{*}$ for the polar body of $K$ with respect to the unit sphere centered at the origin; i.e.,

$$
\rho\left(K^{*}, u\right)=h(K, u)^{-1} .
$$

For the polar bodies of $\Pi\left(K_{1}, \ldots, K_{n-1}\right), \Pi_{i} K$, and $\Pi K$ we will write $\Pi^{*}\left(K_{1}, \ldots, K_{n-1}\right), \Pi_{i}^{*} K$, and $\Pi^{*} K$, rather than $\left(\Pi\left(K_{1}, \ldots, K_{n-1}\right)\right)^{*},\left(\Pi_{i} K\right)^{*}$, and $(\Pi K)^{*}$.

An important inequality involving polars of projection bodies was obtained by Petty [26] (see [24] for an alternate proof) by using an inequality for centroid bodies [25]. The Petty projection inequality states that for a convex body $K$ in $\mathbb{R}^{n}$,

$$
V(K)^{n-1} V\left(\Pi^{*} K\right) \leqslant\left(\omega_{n} / \omega_{n-1}\right)^{n},
$$

with equality if and only if $K$ is an ellipsoid. It was shown in [24] that either directly, or as a consequence of the Petty projection inequality, one can obtain the following general projection inequality for the convex bodies $K_{1}, \ldots, K_{n-1}$ in $\mathbb{R}^{n}$ :

$$
V\left(K_{1}\right) \cdots V\left(K_{n-1}\right) V\left(\Pi^{*}\left(K_{1}, \ldots, K_{n-1}\right)\right) \leqslant\left(\omega_{n} / \omega_{n-1}\right)^{n},
$$

with equality if and only if the $K_{i}$ are homothetic ellipsoids. For the case where the $K_{i}$ are equal, (5.6) will, of course, reduce to (5.5). If $K_{1}=\cdots=K_{n-i-1}=K$ and $K_{n-i}=\cdots=K_{n-1}=B$ for $0<i<n-1$, then (5.6) reduces to

$$
\omega_{n-1}^{n} V(K)^{n-i-1} V\left(\Pi_{i}^{*} K\right) \leqslant \omega_{n}^{n-i},
$$

with equality if and only if $K$ is a ball. It was shown in [24] that (5.7) can be viewed as an extension of the well-known inequalities between the volume and the projection measures of a convex body. A stronger inequality than (5.7) will soon be given.

The following preliminary result will be needed.

(5.8) Proposition. If $K_{1}, \ldots, K_{n-1}$ are convex bodies in $\mathbb{R}^{n}$, then

$$
v\left(\left[K_{1}, \ldots, K_{n-1}\right]^{u}\right)=v\left(K_{1}^{u}, \ldots, K_{n-1}^{u}\right) .
$$


Proof. The $(n-1)$-dimensional mixed volume of $K_{1}^{u}, \ldots, K_{n-1}^{u}$ is related to an $n$-dimensional mixed volume involving $K_{1}, \ldots, K_{n-1}$ (see, for example, [7, p. 45]) by

$$
v\left(K_{1}^{u}, \ldots, K_{n-1}^{u}\right)=n V\left(K_{1}, \ldots, K_{n-1},\langle u\rangle\right)
$$

where $\langle u\rangle$ denotes the closed line segment $\{\lambda u$ : $|\lambda| \leqslant 1 / 2\}$. From (5.9) it follows that for a convex body $C$ in $\mathbb{R}^{n}$ one has

$$
v\left(C^{u}\right)=n V_{1}(C,\langle u\rangle)
$$

If we take $\left[K_{1}, \ldots, K_{n-1}\right]$ for $C$ we get

$$
v\left(\left[K_{1}, \ldots, K_{n-1}\right]^{u}\right)=n V_{1}\left(\left[K_{1}, \ldots, K_{n-1}\right],\langle u\rangle\right) .
$$

To complete our proof we need merely observe that, by (3.4), the right side of this equation is the same as the right side of (5.9).

For the case where $K_{1}=\cdots=K_{n-i-1}=K$ and $K_{n-i}=\cdots=K_{n-1}=B$, Proposition (5.8) becomes

$$
v\left([K]_{i}^{u}\right)=w_{i}\left(K^{u}\right) .
$$

As an immediate consequence we obtain

(5.10) Proposition. A convex body $K$ in $\mathbb{R}^{n}$ is of constant $(n-i-1)$-girth if and only if $[K]_{i}$ is of constant brightness.

Proposition (5.10) was obtained by Firey [17] (for $i=n-2$ ).

From (5.1), (5.2), and Proposition (5.8) it follows that the projection body of the mixed body $\left[K_{1}, \ldots, K_{n-1}\right]$ is just the mixed projection body of $K_{1}, \ldots, K_{n-1}$ :

(5.11) Proposition. If $K_{1}, \ldots, K_{n-1}$ are convex bodies in $\mathbb{R}^{n}$, then

$$
\Pi\left[K_{1}, \ldots, K_{n-1}\right]=\Pi\left(K_{1}, \ldots, K_{n-1}\right) .
$$

We note that Proposition (5.11), in conjunction with Theorem (4.1), is yet another way that one can obtain the general version of the Petty projection inequality (5.6) from the Petty projection inequality (5.5).

For the case where $K_{1}=\cdots=K_{n-i-1}=K$ and $K_{n-i}=\cdots=K_{n-1}=B$, Proposition (5.11) states that

$$
\Pi[K]_{i}=\Pi_{i} K .
$$

The right inequality of Theorem (4.3) is

$$
V\left([K]_{i}\right)^{(n-1) / n} \leqslant \omega_{n}^{-1 / n} W_{i+1}(K) .
$$

The Petty projection inequality (5.5), when combined with (5.12), immediately yields the stronger result

$$
V\left([K]_{i}\right)^{(n-1) / n} \leqslant\left(\omega_{n} / \omega_{n-1}\right) V\left(\Pi_{i}^{*} K\right)^{-1 / n} .
$$

To see that (5.13) is a stronger inequality, and for later use as well, we recall the Kubota formulas (see, for example, [20, p. 184]):

$$
\omega_{n-1} W_{i+1}(K)=\frac{1}{n} \int_{S^{n-1}} w_{i}\left(K^{u}\right) d S(u) .
$$

The Kubota formulas can be derived easily from (1.4) and (5.9). 
Consider now the $p$-mean of the $(n-i-1)$-girth function of $K$, which, for real $p \neq 0$, is

$$
\left[\frac{1}{n \omega_{n}} \int_{S^{n-1}} w_{i}\left(K^{u}\right)^{p} d S(u)\right]^{1 / p} .
$$

For $p=-\infty, 0$, or $\infty$ one can, in the standard way, define the $p$-means by a limiting process (see [19] for details).

From Jensen's or the Hölder inequality [19, p. 140] it follows that unless $K$ is a body of constant $(n-i-1)$-girth, the $p$-means of the $(n-i-1)$-girth function of $K$ are monotone increasing in $p$.

From (5.3) and (5.4) one has

$$
\rho\left(\Pi_{i}^{*} K, u\right)=w_{i}\left(K^{u}\right)^{-1} .
$$

Thus, from the polar coordinate formula for $n$-dimensional volume, we have

$$
V\left(\Pi_{i}^{*} K\right)=\frac{1}{n} \int_{S^{n-1}} w_{i}\left(K^{u}\right)^{-n} d S(u) .
$$

This, when combined with the fact that the $p$-means of the $(n-i-1)$-girth function of $K$ are nondecreasing in $p$, shows that, for $p>-n$,

$$
\omega_{n}^{1 / n} V\left(\Pi_{i}^{*} K\right)^{-1 / n} \leqslant\left[\frac{1}{n \omega_{n}} \int_{S^{n-1}} w_{i}\left(K^{u}\right)^{p} d S(u)\right]^{1 / p},
$$

with equality if and only if $K$ is of constant $(n-i-1)$-girth.

If we look at (5.14), and (5.15) with $p=1$, it is easy to see that inequality (5.13) is indeed stronger than the right inequality of Theorem (4.3).

We note, as an aside, that the Petty projection inequality, in conjunction with Proposition (5.8), will give a better upper bound (in light of [24, Corollary (5.3)]) for $V\left(\left[K_{1}, \ldots, K_{n-1}\right]\right)^{n-1}$ than $V^{n}\left(K_{1}, \ldots, K_{n-1}, B\right) / \omega_{n}$. Specifically, one gets

$$
V\left(\left[K_{1}, \ldots, K_{n-1}\right]\right)^{n-1} \leqslant\left(\frac{\omega_{n}}{\omega_{n-1}}\right)^{n}\left[\frac{1}{n} \int_{S^{n-1}} v\left(K_{1}^{u}, \ldots, K_{n-1}^{u}\right)^{-n} d S(u)\right]^{-1} .
$$

If (5.13) is combined with the left inequality of Theorem (4.3) we get

(5.16) THEOREM. If $K$ is a convex body in $\mathbb{R}^{n}$ and $0<i<n-1$, then

$$
\omega_{n}^{i / n} W_{i}(K)^{n-i-1} V\left(\Pi_{i}^{*} K\right)^{(n-i) / n} \leqslant\left(\omega_{n} / \omega_{n-1}\right)^{n-i},
$$

with equality if and only if $K$ is a ball.

It is easy to see (from the inequalities between the volume and projection measures of a convex body) that this is a stronger result than the general projection inequality (5.7).

We recall that the inequalities between consecutive projection measures of a convex body (see, for example, Theorem 4.3) are

$$
\left(W_{i}(K) / \omega_{n}\right)^{(n-i-1) /(n-i)} \leqslant W_{i+1}(K) / \omega_{n} .
$$


If (5.15) is combined with Theorem (5.16) we get

(5.18) CoROllaRY. If $K$ is a convex body in $\mathbb{R}^{n}, 0<i<n-1$, and $p \geqslant-n$, then

$$
\omega_{n-1}\left(W_{i}(K) / \omega_{n}\right)^{(n-i-1) /(n-i)} \leqslant\left[\frac{1}{n \omega_{n}} \int_{S^{n-1}} w_{i}\left(K^{u}\right)^{p} d S(u)\right]^{1 / p},
$$

with equality if and only if $K$ is a ball.

In light of (5.14), we see that for $p=1$, this reduces to (5.17), while for $-n \leqslant p<1$, the inequalities of Corollary (5.18) are strengthened forms of the inequalities between consecutive projection measures of a convex body (5.17).

The classical inequalities between the projection measures of a convex body are easily obtained by repeated applications of (5.17). They state that for a convex body $K$ in $\mathbb{R}^{n}$, and for $0 \leqslant i \leqslant j<n-1$ :

$$
\left(W_{i}(K) / \omega_{n}\right)^{(n-j-1) /(n-i)} \leqslant W_{j+1}(K) / \omega_{n} .
$$

From Corollary (5.18) we have

(5.20) CoRollary. If $K$ is a convex body in $\mathbb{R}^{n}, 0<i \leqslant j<n-1$, and $p \geqslant-n$, then

$$
\omega_{n-1}\left(\frac{W_{i}(K)}{\omega_{n}}\right)^{(n-j-1) /(n-i)} \leqslant\left[\frac{1}{n \omega_{n}} \int_{S^{n-1}} w_{j}\left(K^{u}\right)^{p} d S(u)\right]^{1 / p},
$$

with equality if and only if $K$ is a ball.

The case $i=j$ is, of course, just Corollary (5.18), while the cases where $i<j$ follow directly from Corollary (5.18) if we use (5.19).

From (5.14) it follows that the case $p=1$ of Corollary (5.20) reduces to the known inequality between the projection measures of a convex body (5.19), while the cases of Corollary (5.20) with $-n \leqslant p<1$ are strengthened forms.

The case $i=0$ of Corollary (5.20) is found in [24].

6. Mixed projection integrals. For convex bodies $K_{1}, \ldots, K_{n}$ in $\mathbb{R}^{n}$ and an integer $r$ such that $0 \leqslant r<n$, the $r$ th mixed projection integral of $K_{1}, \ldots, K_{n}$, $I_{r}\left(K_{1}, \ldots, K_{n}\right)$, is defind by

$$
I_{r}\left(K_{1}, \ldots, K_{n}\right)=\frac{\omega_{n}^{n-r-2}}{n \omega_{n-1}^{n}} \int_{S^{n-1}} w_{r}\left(K_{1}^{u}\right) \cdots w_{r}\left(K_{n}^{u}\right) d S(u) .
$$

In [23] for $r=0$, and in [24] for $0 \leqslant r<n-1$, it was shown that

$$
\left[V\left(K_{1}\right) \cdots V\left(K_{n}\right)\right]^{n-r-1} \leqslant I_{r}\left(K_{1}, \ldots, K_{n}\right)^{n},
$$

with equality if and only if the $K_{i}$ are balls. For the cases $0<r<n-1$, a better result is now possible. 
A simple application of the Hölder inequality [19, p. 140] will yield (see [24, p. 104])

$$
\left[\frac{1}{n} \int_{S^{n-1}}\left[w_{r}\left(K_{1}^{u}\right) \cdots w_{r}\left(K_{n}^{u}\right)\right]^{-1} d S(u)\right]^{n} \leqslant V\left(\Pi_{r}^{*} K_{1}\right) \cdots V\left(\Pi_{r}^{*} K_{n}\right),
$$

with equality if and only if the $(n-r-1)$-girth functions of the $K_{i}$ are proportional to each other.

From the Schwarz or Hölder inequality one gets

$$
n \omega_{n}^{n-r} \leqslant \omega_{n-1}^{n} I_{r}\left(K_{1}, \ldots, K_{n}\right) \int_{S^{n-1}}\left[w_{r}\left(K_{1}^{u}\right) \cdots w_{r}\left(K_{n}^{u}\right)\right]^{-1} d S(u) .
$$

If (6.2), (6.3) and Theorem (5.16) are combined, the result is

(6.4) THEOREM. If $K_{1}, \ldots, K_{n}$ are convex bodies in $\mathbb{R}^{n}$ and $0<r<n-1$, then

$$
\left[W_{r}\left(K_{1}\right) \cdots W_{r}\left(K_{n}\right)\right]^{n-r-1} \leqslant \omega_{n}^{r(n-r-1)} I_{r}\left(K_{1}, \ldots, K_{n}\right)^{n-r},
$$

with equality if and only if the $K_{i}$ are balls.

That the inequalities of Theorem (6.4) are improvements of (6.1) follows immediately from the known inequalities between the volume and projection measures of a convex body, viz. (5.19).

It is possible to obtain more general results by considering more general mixed projection integrals. For convex bodies $K_{1}, \ldots, K_{n}$ in $\mathbb{R}^{n}$ and nonnegative integers $r_{1}, \ldots, r_{n}<n-1$, we define the mixed projection integral $J\left(K_{1}, r_{1} ; \ldots ; K_{n}, r_{n}\right)$ by

$$
J\left(K_{1}, r_{1} ; \ldots ; K_{n}, r_{n}\right)=\frac{1}{n \omega_{n}} \int_{S^{n-1}} w_{r_{1}}\left(K_{1}^{u}\right) \cdots w_{r_{n}}\left(K_{n}^{u}\right) d S(u) .
$$

For convenience we introduce the constant $\beta\left(r_{1}, \ldots, r_{n}\right)$, defined by

$$
\beta\left(r_{1}, \ldots, r_{n}\right)=\omega_{n-1}^{-n} \prod_{i=1}^{n} \omega_{n}^{\left(n-r_{i}-1\right) /\left(n-r_{i}\right)} .
$$

In the same way that Theorem (6.4) is established, one gets

(6.4*) THEOREM. If $K_{1}, \ldots, K_{n}$ are convex bodies in $\mathbb{R}^{n}$ and $r_{1}, \ldots, r_{n}$ are nonnegative integers less than $n-1$, then

$$
\prod_{i=1}^{n} W_{r_{i}}\left(K_{i}\right)^{\left(n-r_{i}-1\right) /\left(n-r_{i}\right)} \leqslant \beta\left(r_{1}, \ldots, r_{n}\right) J\left(K_{1}, r_{1} ; \ldots ; K_{n}, r_{n}\right)
$$

with equality if and only if the $K_{i}$ are balls.

Clearly, Theorem (6.4) is the special case of Theorem $\left(6.4^{*}\right)$ where all the $r_{i}$ are equal.

\section{REFERENCES}

1. A. D. Aleksandrov, Extension of certain concepts in the theory of convex bodies, Mat. Sb. (N.S.) 2 (1937), 947-972. (Russian)

2. __ New inequalities between mixed volumes and their applications, Mat. Sb. (N.S.) 2 (1937), 1205-1238. (Russian)

3. Extension of two theorems of Minkowski on convex polyhedra to arbitrary convex bodies, Mat. Sb. (N.S.) 3 (1938), 27-46. (Russian)

4. ___ Mixed discriminants and mixed volumes, Mat. Sb. (N.S.) 3 (1938), 227-251. (Russian)

5. __ On the surface area function of a convex body, Mat. Sb. (N.S.) 6 (1939), 167-174. (Russian) 
6. E. D. Bolker, A class of convex bodies, Trans. Amer. Math. Soc. 145 (1969), 323-345.

7. T. Bonnesen and W. Fenchel, Theorie der konvexen Körper, Springer-Verlag, Berlin, 1934.

8. Ju. D. Burago and V. A. Zalgaller, Geometric inequalities, "Nauka”, Leningrad. Otdel., Leningrad, 1980. (Russian)

9. H. Busemann, Convex surfaces, Interscience, New York, 1958.

10. G. D. Chakerian, Sets of constant relative width and constant relative brightness, Trans. Amer. Math. Soc. 129 (1967), 26-37.

11. The mean volume of boxes and cylinders circumscribed about a convex body, Israel J. Math. 12 (1972), 249-256.

12. Isoperimetric inequalities for the mean width of a convex body, Geom. Dedicata 1 (1973), 356-362.

13. Geometric inequalities for plane convex bodies, Canad. Math. Bull. 22 (1979), 9-16.

14. G. D. Chakerian and J. R. Sangwine-Yager, A generalization of Minkowski's inequality for plane convex sets. Geom. Dedicata 8 (1979), 437-444.

15. W. Fenchel and B. Jessen, Mengenfunktionen und konvexe Körper, Danske Vid. Selskab. Mat.-Fys. Medd. 16 (1938), 1-31.

16. W. J. Firey and B. Grünbaum, Addition and decomposition of convex polytopes, Israel J. Math. 2 (1964), 91-100.

17. W. J. Firey, Blaschke sums of convex bodies and mixed bodies, Proc. Colloq. Convexity (Copenhagen 1965), Københavns Univ. Mat. Inst., 1967, pp. 94-101.

18. B. Grübaum, Convex polytopes, Interscience, New York, 1967.

19. G. H. Hardy, J. E. Littlewood and G. Pólya, Inequalities, Cambridge Univ. Press, Cambridge, 1934.

20. K. Leichtweiss, Konvexe Mengen, Springer-Verlag, Berlin, 1980.

21. E. Lutwak, Width-integrals of convex bodies, Proc. Amer. Math. Soc. 53 (1975), 435-439.

22. __ Mixed width-integrals of convex bodies, Israel J. Math. 28 (1977), 249-253.

23. __ A general isepiphanic inequality, Proc. Amer. Math. Soc. 90 (1984), 415-421.

24. __ Mixed projection inequalities, Trans. Amer. Math. Soc. 287 (1985), 91-106.

25. C. M. Petty, Centroid surfaces, Pacific J. Math. 11 (1961), 1535-1547.

26. Isoperimetric problems, Proc. Conf. Convexity and Combinatorial Geometry, Univ. of Oklahoma (June 1971), 1972, pp. 26-41.

27. R. Schneider, Über Tangentialkörper der Kugel, Manuscripta Math. 23 (1978), 269-278.

28. ___ Boundary structure and curvature of convex bodies, Contributions to Geometry (J. Tölke and J. M. Wills, eds.), Birkhäser Verlag, Basel, 1979, pp. 1-59.

29. __ On the Aleksandrov-Fenchel inequality, Discrete Geometry and Convexity (J. E. Goodman et al., eds.), Ann. N. Y. Acad. Sci. 440 (1985), 132-141.

30. R. Schneider and W. Weil, Zonoids and related topics, Convexity and Its Applications (P. M. Gruber and J. M. Wills, eds.), Birkhäuser Verlag, Basel, 1983, pp. 296-317.

Department of Mathematics, Polytechnic Institute of New York, Brooklyn, New York 11201 